\title{
Dust Mobilization and Transport Modeling for Loss of Vacuum Accidents
}

\section{$8^{\text {th }}$ International Symposium on Fusion Nuclear Technology}

P. W. Humrickhouse

J. P. Sharpe

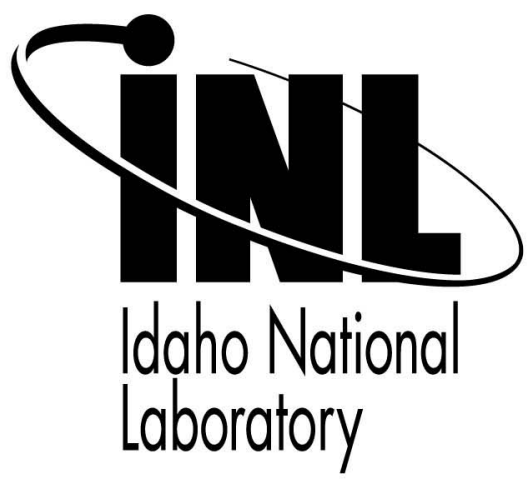

\section{October 2007}

This is a preprint of a paper intended for publication in a journal or proceedings. Since changes may be made before publication, this preprint should not be cited or reproduced without permission of the author. This document was prepared as an account of work sponsored by an agency of the United States Government. Neither the United States Government nor any agency thereof, or any of their employees, makes any warranty, expressed or implied, or assumes any legal liability or responsibility for any third party's use, or the results of such use, of any information, apparatus, product or process disclosed in this report, or represents that its use by such third party would not infringe privately owned rights. The views expressed in this paper are not necessarily those of the United States Government or the sponsoring agency. 


\title{
Dust mobilization and transport modeling for loss of vacuum accidents
}

\author{
P.W. Humrickhouse ${ }^{a, b, *}$, J.P. Sharpe ${ }^{b}$ \\ a Department of Engineering Physics, University of Wisconsin, 1500 Engineering Drive, Madison, WI 53706, United States \\ ${ }^{\mathrm{b}}$ Fusion Safety Program, Idaho National Laboratory, P.O. Box 1625. MS 3840, Idaho Falls, ID 83415, United States
}

Keywords:

Dust mobilization

Tokamak dust

Aerosol dynamics

Granular flow

Soil mechanics

Constitutive modeling

\begin{abstract}
A B S T R A C T
We develop a general continuum fluid dynamic model for dust transport in loss of vacuum accidents in fusion energy systems. The relationship between this general approach and established particle transport methods is clarified, in particular the relationship between the seemingly disparate treatments of aerosol dynamics and Lagrangian particle tracking. Constitutive equations for granular flow are found to be inadequate for prediction of mobilization, as these models essentially impose a condition of flow from the outset. Experiments confirm that at low shear, settled dust piles behave more like a continuum solid, and suitable solid models will be required to predict the onset of dust mobilization.
\end{abstract}

\section{Introduction}

Erosion of plasma facing surfaces in tokamaks is known to result in accumulation of dust inside the vacuum vessel. In ITER, accumulation of large quantities of dust presents several safety hazards; these include dust explosions, hydrogen production through beryllium-steam reactions, and release of radioactive/toxic particles. In all cases, the concern is that vessel filling during a loss of vacuum accident (LOVA) may result in the mobilization of settled dust as pressures equilibrate. Here, we discuss the applicability of various dust transport models to this problem, and in particular seek a model for prediction of the extent of dust mobilization for a given set of flow conditions.

\section{Particle transport regimes}

The aforementioned accident scenarios involve large quantities (kilograms) of dust. This precludes a treatment of individual particles, so we treat the particles as a continuum fluid. The momentum of this continuum "particle fluid", as with any other, is given by the Navier-Stokes equations:

$\frac{\mathrm{D} \underline{\underline{v}}}{\mathrm{D} t}=-\frac{1}{\rho} \nabla p+\frac{1}{\rho} \nabla \cdot \underline{\underline{\boldsymbol{T}}}+\underline{A}$.

* Corresponding author at: Fusion Safety Program, Idaho National Laboratory, P.O. Box 1625, MS 3840, Idaho Falls, ID 83415, United States. Tel.: +1 2085267496 ; fax: +1 2085262930 .

E-mail addresses: paul.humrickhouse@inl.gov. paul@humrickhouse.com (P.W. Humrickhouse).
Here $v$ is the particle velocity; $\rho$ is the bulk particle density, $p$ the particle phase pressure; $\mathbf{T}$ is the viscous stress tensor; and $A$ the acceleration due to body forces. The four terms are due to inertial, pressure, viscous, and body forces, respectively. First we consider the limiting case in which interactions between particles are negligible. This suggests we may safely ignore the particle interaction ("viscous") terms, and eliminate the tensor T. Furthermore, as an ensemble of non-interacting particles, the ideal gas law applies; so the pressure term may be rewritten as follows:

$\frac{\mathrm{D} \underline{\underline{v}}}{\mathrm{D} t}=-\frac{1}{\rho} \nabla n k T+\underline{A}$.

Identifying the bulk density as the particle concentration $\mathrm{n}$ times the individual particle mass $m$, and assuming constant temperature,

$\frac{\mathrm{D} \underline{v}}{\mathrm{D} t}=-\frac{k T}{m} \frac{\nabla n}{n}+\underline{A}$

In cases of practical interest, the particle fluid does not exist as a single phase, but is suspended in another fluid. We treat the two phases as interpenetrating continua, where the body force term in the particle momentum equation is due to drag from the suspending fluid. Now we further restrict ourselves to low Stokes numbers, where

$\mathrm{Stk}=\frac{\tau U}{L}$

and $U$ and $L$ are the characteristic velocity and length scale of the flow, and $\tau$ is the spherical particle relaxation time given by

$\tau=\frac{\rho_{\mathrm{s}} d^{2}}{24 \mu}$ 
for fluid viscosity $\mu$, and particle diameter $d$, and solid material density $\rho_{\mathrm{s}}$. As a ratio of inertial to viscous (drag) forces, low Stokes numbers imply negligible inertia, and we discard the inertial terms in Eq. (3) accordingly. The drag force is given by Stokes" law, and

$\underline{A}=\frac{\underline{u}-\underline{v}}{\tau}$

for the particle velocity $v$ relative to the fluid velocity $u$. The momentum equation simplifies to

$\frac{\tau k T}{m} \nabla n=n(\underline{u}-\underline{v})$.

The particle velocity can be solved for explicitly; recognizing the Einstein diffusion coefficient $D[1]$,

$D=\frac{\tau k T}{m}$,

the particle flux is given by

$n \underline{v}=n \underline{u}-D \nabla n$.

Using this expression in the continuity equation,

$\frac{\partial n}{\partial t}+\nabla \cdot(n \underline{v})=0$,

we arrive at the 'aerosol dynamic equation' as used in aerosol transport phenomena [2]:

$\frac{\partial n}{\partial t}+\nabla \cdot(n \underline{u})=D \nabla^{2} n$.

Thus, the aerosol dynamic equation is a special case of a general particle fluid described by the Navier-Stokes equations, in which particle interactions and particle inertia are neglected.

If one neglects diffusion (pressure) rather than inertia (i.e. only inertia and drag force terms are retained), the resulting system is hyperbolic, and its characteristics are the Lagrangian equations of motion [3]. Thus, aerosol dynamics and Lagrangian particle tracking treat two opposite dilute flow regimes: purely diffusive and purely inertial, respectively. Any problem in which both effects may be important (but particles are still non-interacting) should solve the general case that accounts for both (Eq. (3)).

The problem of dust transport in a loss of vacuum accident cannot be simplified to either of the two cases above a priori. Here, flow conditions will vary widely in both space and time. The particle relaxation time varies as the particle diameter squared, and this diameter may span several orders of magnitude. Furthermore, local velocity gradients $(U / L)$ will be high in regions near the breach, but lower elsewhere, and will everywhere decrease in time as the vessel fills with air. Rather than selecting a model that may restrict our ability to consider certain particle sizes, materials, and flow regimes, we elect to treat the general case.

Furthermore, the initial condition of the vessel filling problem violates the very first assumption of both previously described models; here dust will be settled into relatively dense layers and piles in which particles contact each other; the nature of these particle interactions will determine the extent of dust mobilization during loss of vacuum.

\section{Constitutive equations for granular materials}

Since particle interactions are clearly important, at least in the initial stages of a LOVA, a constitutive equation is required to specify the viscous stress tensor $\mathbf{T}$. At intermediate particle volume fractions, particles undergo binary collisions, and closures are determined from kinetic theory [4]. At higher particle volume fractions and lower rates of shear, particles develop enduring contacts and their interaction is best described as frictional. We adopt the following [5,6]:

$\boldsymbol{T}=\frac{\sqrt{2} P \sin \phi}{\sqrt{\boldsymbol{S}: \boldsymbol{S}}} \boldsymbol{S}$

where $\mathbf{S}$ is the deviatoric strain rate tensor,

$\boldsymbol{S}=\frac{1}{2}\left(\nabla \underline{v}-(\nabla \underline{v})^{\mathrm{T}}\right)-\frac{1}{3}(\nabla \cdot \underline{v}) \boldsymbol{I}$,

$P$ is the particle phase pressure, and $\phi$ is the yield angle. It is useful to cast Eq. (12) in terms of a "frictional viscosity", where

$\mathbf{T}=\mu_{\mathrm{f}} \mathrm{S}$

and

$\mu_{\mathrm{f}}=\frac{\sqrt{2} P \sin \phi}{\sqrt{\boldsymbol{S}: \boldsymbol{S}}}$

$P$ is given now by the empirical expression $[6,7,8]$

$P=\frac{0.05}{v} \frac{\left(v-v_{\min }\right)^{2}}{\left(v_{\max }-v\right)^{5}}$.

Eq. (12) captures the zero order dependence of stress on the strain rate that is observed for granular materials, and assumes a state of incipient yield; i.e. the shear stress is at a critical fraction of the normal stress, determined by the Coulomb yield criterion for cohesionless materials. For shear stress $\tau$, normal stress $\sigma$, and yield angle $\phi$,

$\tau=\sigma \tan \phi$.

The empirical expression for pressure (Eq. (16)) reflects the fact that the pressure becomes large (i.e. diverges) as the particle volume fraction $(\nu)$ approaches a maximum limit, typically taken to be "random close packing", which for spheres is $\sim 0.63$. Frictional effects are assumed to be zero below a minimum particle volume fraction, typically taken to be 0.5 .

\section{Fluent modeling of dust mobilization}

The frictional viscosity model described in the previous section has been implemented in the commercial computational fluid dynamics code Fluent to model dust mobilization in a horizontal pipe. Here, we examine laminar pipe flows in an attempt to establish a threshold for mobilization, and to eliminate characterization of complex flow patterns and turbulence as confounding variables in determining that threshold. In the model, a fully developed profile enters a $19 \mathrm{~mm}$ diameter pipe and interacts with a settled dust pile at some point downstream.

The mobilization fraction is defined as the fraction of the total dust that remains in the initially defined volume of the dust pile, and is monitored as a function of time. The frictional viscosity model is compared with a base case in which no particle interactions are modeled, i.e. the particle stress tensor is zero. While the details vary according to the initial conditions and particle properties, results fall into one of two categories: (1) the rate of mobilization decreases, but only slightly, with the addition of frictional viscosity, and (2) the code fails due to divergence of the frictional pressure or viscosity. In the first case (see Fig. 1), we suspect that the rapid mobilization is unphysical. The second (Fig. 2) is not a numerical problem, but reflects the nature of the constitutive model. The viscosity diverges both as the particle volume fraction approaches a maximum (Eq. (16)), and as the shear rate goes to zero (Eq. (12)). The second of these is particularly problematic in this case, as the dust pile is initially at rest, with velocity and velocity gradients (shear rate) everywhere zero. In practice this is commonly avoided 


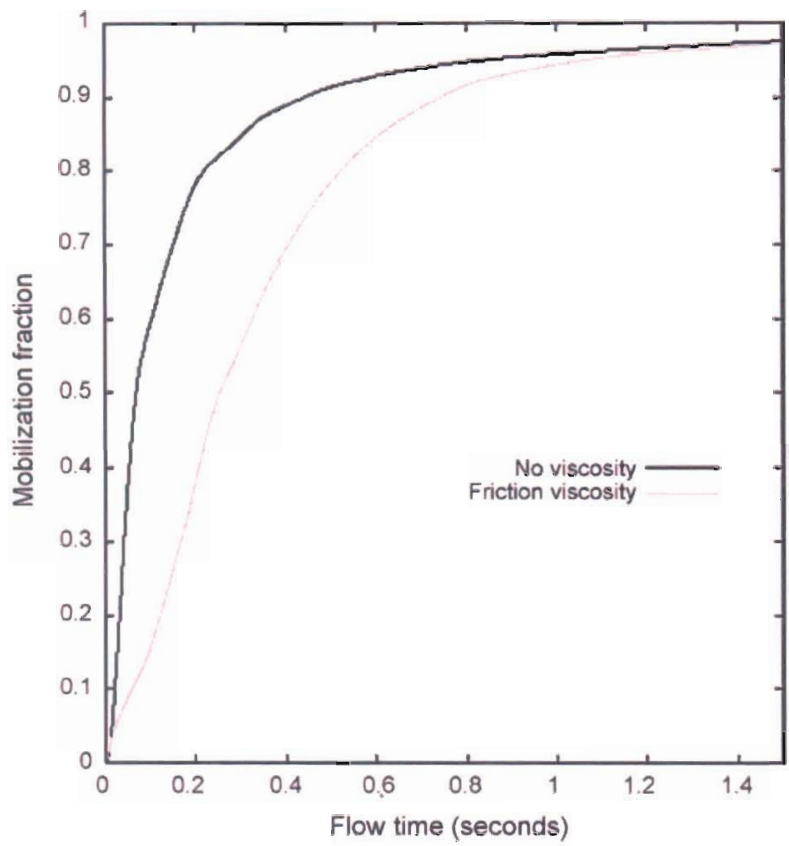

Fig. 1. Predicted mobilization fraction as a function of time for $5 \mu \mathrm{m}$ graphite dust subjected to flow of air at $R e=500$. The addition of a simplified frictional viscosity model has minimal effect on the rapid mobilization. Here, $\mu_{\mathrm{f}}=P \sin \phi$, and $P$ is given by Eq. (16).

by adding a constant to the scalar product of the deviatoric strain rate tensor $\mathbf{S : S}[6,9]$, e.g.

$\mu_{\mathrm{f}}=\frac{\sqrt{2} P \sin \phi}{\sqrt{S: S+C}}$,

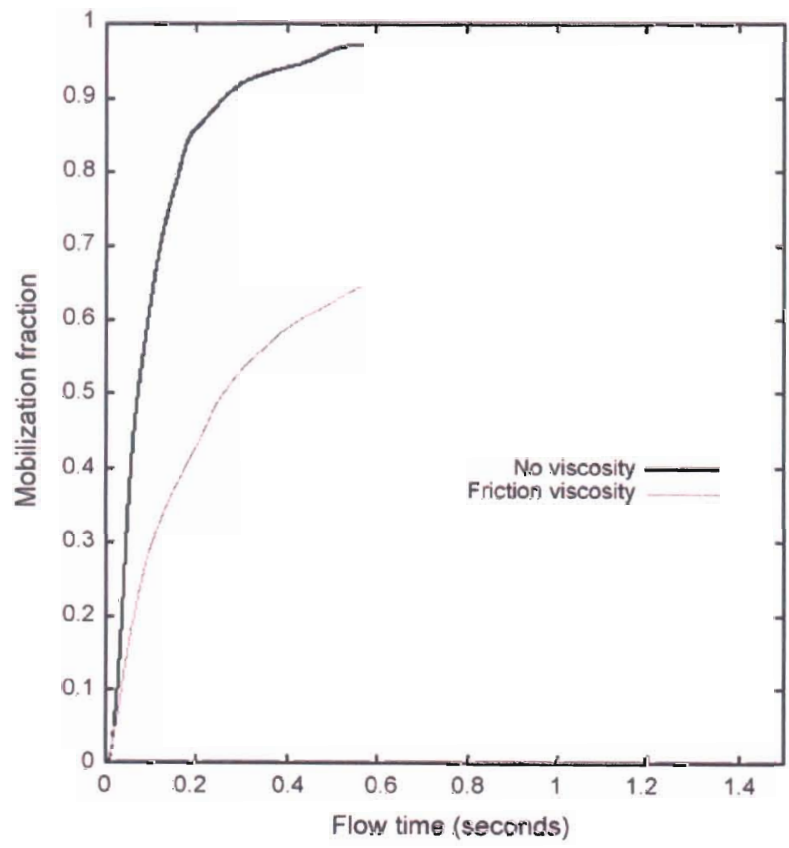

Fig. 2. Predicted mobilization fraction as a function of time for $65 \mu \mathrm{m}$ stainless steel dust subjected to flow of air at $\operatorname{Re}=930$, up to the point of divergence at $t \sim 0.56 \mathrm{~s}$. The frictional viscosity model used is described in Section 3. or by limiting the maximum allowable value for viscosity, as is done in Fluent [8]. But here the zero-order dependence on shear rate is lost, and the implication is that fluid constitutive models of this type cannot predict stationary behavior of granular materials, even when this is the physical reality. This is an acknowledged failing of such models [6]. Divergence at maximum volume fraction and zero strain rate are characteristic of a transition from fluid to solidlike behavior, but the behavior of such a solid under shear cannot be characterized within the context of fluid dynamics. Indeed, the assumption that the granular material is at the point of yield everywhere was assumed in the development of the frictional viscosity model [5]. We seek a predictive model, which allows determination of stresses in a solid-like granular material, which may or may not satisfy the yield criterion. If yield occurs, the two-phase fluid dynamics problem may be undertaken as described previously to model subsequent dust transport; if not, mobilization will not occur - an important conclusion for the safety analysis of LOVAs.

\section{Experiments}

An experimental campaign has begun to investigate the behavior of dust piles at low shear. The experiment has the same configuration modeled in Fluent; a $19 \mathrm{~mm}$ diameter horizontal pipe, with an approximately $3 \mathrm{~m}$ entrance length upstream of a dust pile to establish fully developed flow. While more sophisticated diagnostics are planned to measure the extent of dust mobilization and the ensuing transport, qualitative observations alone offer valuable insight into the behavior of such materials at low shear. Two materials have been tested thus far; 316 stainless steel of $65 \mu \mathrm{m}$ diameter (Fig. 3), and graphite of $4 \mu \mathrm{m}$ diameter (Fig. 4). The latter is a size and material characteristic of dust sampled from tokamaks [10]. The larger stainless steel better approximates the ideal "cohesionless" granular material, an important intermediate step in the evaluation of computational models. Both were subjected to flows of helium, up to a maximum Reynolds number of 930. The behavior of both materials was similar, and consistent with a "yielding solid" description rather than a fluid dynamic description. If the

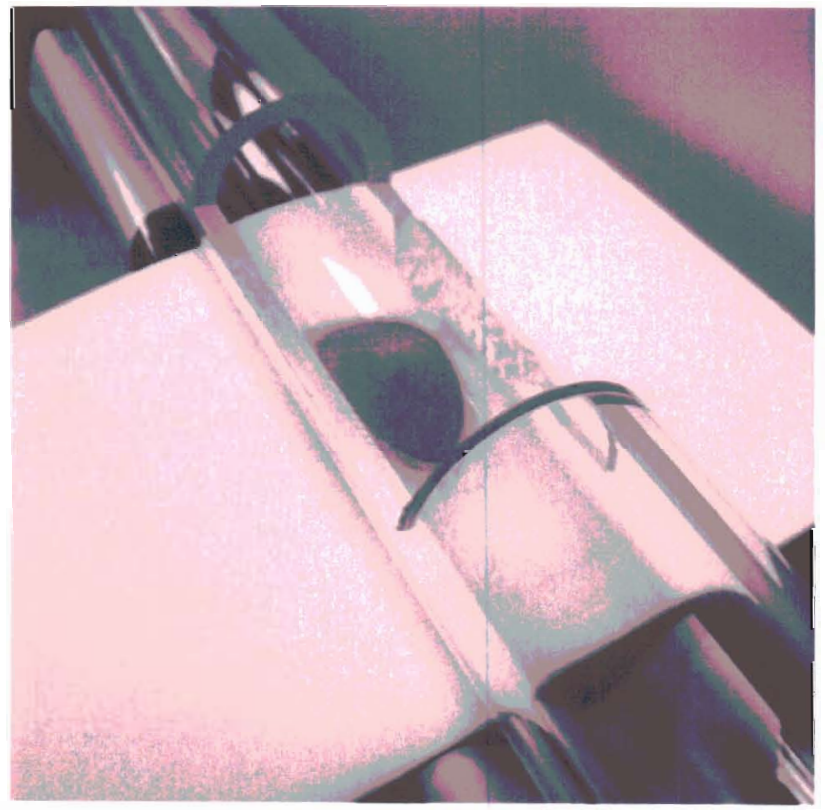

Fig. 3. The pile of $65 \mu \mathrm{m}$ stainless steel dust was not mobilized when subjected to a flow of helium at $\operatorname{Re} \sim 930$. 


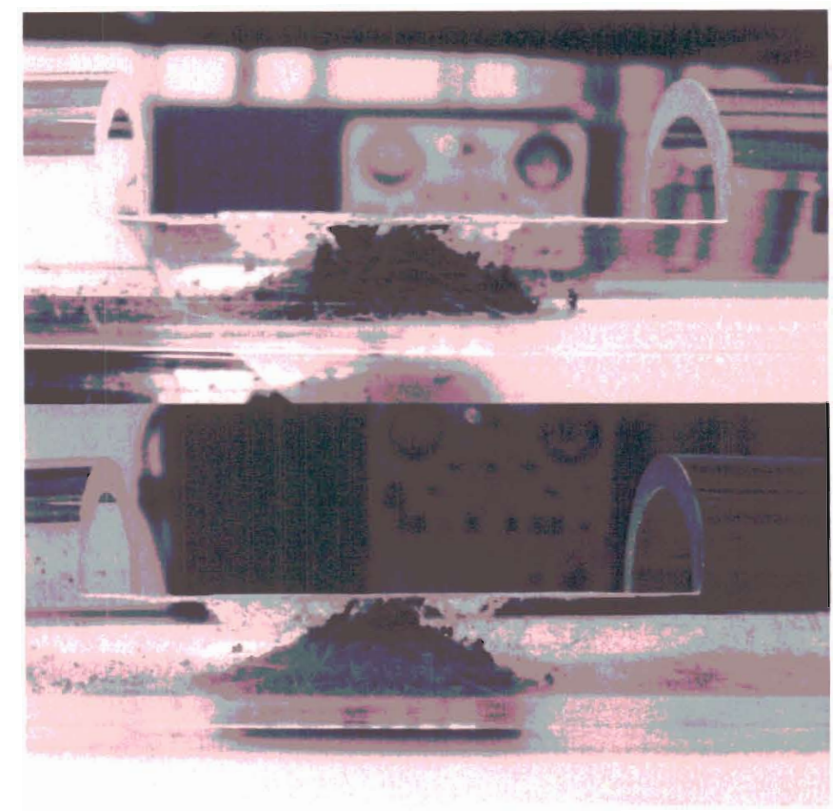

Fig. 4. Graphite dust $(4 \mu \mathrm{m})$ pile before (top) and after (bottom) flow of helium up to $R e \sim 930$. The majority of the pile remained stable at that flow rate. Some material was mobilized, primarily on the upstream (left) side of the pile near the top. Redeposition is visible on the wall of the acrylic tube immediately downstream.

behavior were fluid-like, we would expect to see continual mobilization at constant flow rate, with the rate of mobilization increasing with increasing flow rate. Rather, we observe a dust pile that is stable at Re $\sim 930$, sustaining shear with no observable mobilization as flow continues at that rate. What few changes did occur in the piles were consistent with the idea of yield in a region of the pile; in a few instances, when a small increase in the flow rate was made, a small section of the pile would be mobilized, with the rest of the pile remaining unchanged.

These observations suggest that at rest and for relatively low stresses, the behavior of dust piles is in fact solid; and that mobilization may be identified with yield. But, yield may occur only in small portions of the pile, and mobilization will be partial in this case, with the rest of the pile remaining in a stable configuration.

\section{Conclusions and future work}

We have considered a general continuum fluid approach to modeling dust mobilization and transport. In the limit of non- interacting particles, the general Navier-Stokes equations simplify to those of aerosol dynamics and Lagrangian particle tracking when one chooses to treat only diffusion or inertia, respectively. We consider the general case, with a constitutive equation derived from soil mechanics for dense granular flow. This may be a suitable transport model, but it cannot predict the onset of mobilization because it imposes an initially present state of yield in its development. In other words, the fluid model describes the flow that occurs when the material yields; we require a solid model to calculate stresses in the pile and determine if it yields and the conditions under which yielding occurs.

Future work will evaluate approximate [11] and more rigorous [12] methods for performing such calculations. Experiments will focus on tungsten dusts in light of increased interest in tungsten as a plasma facing material on the ITER divertor as well as the first wall in future devices.

\section{Acknowledgements}

The authors are grateful to Dr. Michael Corradini and Dr. Jason Oakley at the University of Wisconsin for many fruitful discussions, and also to Mr. Lee Cadwallader at Idaho National Laboratory, for constructive comments during preparing of the manuscript.

\section{References}

[1] A. Einstein, The elementary theory of the Brownian motion, Z. Elektrochemie 14 (1908) 235-239.

[2] S.K. Friedlander, Smoke, Dust, and Haze, John Wiley and Sons, New York, 1977.

[3] J. Fernandez de la Mora, D.E. Rosner, Inertial deposition of particles revisited and extended: Eulerian approach to a traditionally Lagrangian problem, PhysicoChemical Hydrodynamics 2 (1981) 1-21.

[4] C.K.K. Lun, S.B. Savage, D.J. Jeffrey, N. Chepurniy, Kinetic theories for granular flow: inelastic particles in Couette flow and slightly inelastic particles in a general flowfield, J. Fluid Mech. 140 (1984) 223-256.

[5] D.G. Schaeffer, Instability in the evolution equations describing incompressible granular flow, J. Diff. Eq. 66 (1987) 19-50.

[6] A. Srivastava, S. Sundaresan, Analysis of a frictional-kinetic model for gasparticle flow, Powder Technol. 129 (2003) 72-85.

[7] P.C. Johnson, R. Jackson, Frictional-collisional constitutive relations for granular materials, with application to plane shearing, J. Fluid Mech. 176 (1987) 67-93.

[8] Personal communication with Fluent support personnel, August 2007.

[9] C. Leppert, D. Dinkler, A Fluid mechanical model for granular flow in silos, Proc. Appl. Math. Mech. 5 (2005) 309-310.

[10] W.J. Carmack, R.A. AnderI, R.J. Pawelko, G.R. Smolik, K.A. McCarthy, Characterization and analysis of dusts produced in three experimental tokamaks: TFTR, DIII-D, and Alcator C-Mod, Fusion Eng. Des. 51-52 (2000) 477 484.

[11] R.M. Nedderman, Statics and Kinematics of Granular Materials, Cambridge University Press, Cambridge, 1992.

[12] Y. Jiang, M. Liu, A brief review of "granular elasticity", Eur. Phys. J. E 22 (2007) $255-260$ 